\title{
Temáticas en construcción: el desarrollo de los estudios LGBT en Costa Rica, 1980-2013
}

\author{
José Daniel Jiménez Bolaños ${ }^{1}$
}

Recepción: 9 de marzo de 2014 / Aprobación: 7 de abril de 2014

\section{Resumen}

Debido a la ausencia de un balance bibliográfico que sistematice el desarrollo que ha experimentado la diversidad sexual como temática de investigación aplicada al caso de Costa Rica, se hizo una revisión con el fin de procesar los textos a través de una base de datos, buscando describir y analizar sus características. Se encontró que durante el periodo analizado se ha incrementado el número de las investigaciones, cuya publicación se da principalmente en Costa Rica, proviniendo la mayor cantidad de aportes de la Escuela de Psicología de la Universidad de Costa Rica. Se evidencia un cambio en las temáticas, pasando de ser prioritario el VIH/SIDA en la década de 1980 a una preocupación por asuntos sobre la legislación, el reconocimiento de parejas del mismo sexo y la transexualidad. Se encontraron vacíos investigativos en torno al análisis de la intersexualidad, la bisexualidad, la intersección de sexualidad con otros dispositivos de poder.

\section{Palabras clave}

Estudios LGBT; balance bibliográfico; diversidad sexual; Costa Rica; desarrollo académico

\section{Abstract}

Due to the lack of a bibliographical balance that shows the development experienced by sexual diversity as a research subject applied to the case of Costa Rica, a search was made with the aim of processing the texts through a data base, looking to describe and analyze their main characteristics. Among the results it was found that there has been an increase in the number of researches during the last three decades, the majority of texts has been published in Costa Rica, most of the contributions come from the School of Psychology in the Universidad de Costa Rica, there has been a change in the themes, going from HIV/AIDS in the eighties, to a multiplicity of topics such as legislation, same sex couples and trans sexuality. There are still pending subject-matters to explore such as intersexuality, bisexuality, the intersection between sexuality and other power structures.

1 Costarricense. Licenciado en Historia por la Universidad de Costa Rica. Investigador independiente. Correo electrónico: josezero6@gmail.com 


\section{Keywords}

LGBT studies; bibliographic balance; sexual diversity; Costa Rica; academic development

\section{Resumo}

Devido à ausência de um balanço bibliográfico para sistematizar o desenvolvimento experimentado a diversidade sexual como tema aplicado ao caso da investigação Costa Rica, a revisão foi feita a fim de processar os textos através de um banco de dados, buscando descrever e analisar suas características. Verificou-se que durante esse período o número das investigações teve um aumento, cujas publicações acontecem principalmente na Costa Rica, onde a maior quantidade de contribuições proveem da Escola de Psicologia da Universidade da Costa Rica. Evidencia-se uma mudança nas temáticas, passando o HIV de ser prioritário, nos anos oitenta, à uma preocupação por assuntos sobre a legislação, o reconhecimento de casais do mesmo gênero e a transexualidade. Foram encontrados vazios investigativos em relação às análises da intersexualidade, a bissexualidade, a intersecção de sexualidade com outros dispositivos de poder, ficando assim tópicos para investigar.

\section{Palavras chave}

Estudos LGBT; balance bibliográfico; diversidade sexual; Costa Rica; desenvolvimento acadêmico

\section{Introducción}

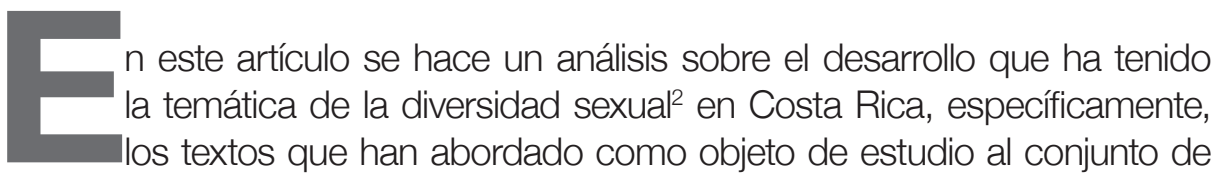

2 Existen varias maneras de conceptualizar a la diversidad sexual, por un lado "designa un conjunto de prácticas, identidades u orientaciones sexuales que en algún momento fueron catalogadas como divergentes de la norma. De ahí que se use como eufemismo para referirse a lo normativamente diferente, incluso a las personas diferentes. Tal es el caso de las enunciaciones sobre la no heterosexualidad en general, donde se nombran sexualidades diversas, hombres diversos o mujeres diversas, tratándose de hombres y de mujeres homosexuales, gays, lesbianas, bisexuales, transgeneristas. Este es el sentido y el uso más frecuente de diversidad sexual. Generalmente, tal acepción desconsidera las jerarquías entre las personas, identidades y prácticas de las que habla, lo que restringe la posibilidad de identificar en este universo relaciones de poder y de discriminación" (Brigeiro, 2012, 83), sin embargo, también "puede referirse a todo tipo de prácticas, identidades, comportamientos, orientaciones y deseos del campo de la sexualidad. En este sentido, diversidad sexual alude tanto a la homosexualidad como a la heterosexualidad; tanto a las prácticas que no configuran necesariamente identidades distintas, como a aquellas que identifican y movilizan a las personas. El término presupone que no existe una única norma de sexualidad y que las normas cambian según el contexto y con el tiempo, subrayando su carácter histórico y cultural. El uso de la expresión diversidad sexual derivado de esta concepción se limita a señalar su variedad, en vez de referirse a un tipo de práctica, identidad u orientación" (Brigeiro, 2012, 84). En ese sentido, aunque en este texto se hable de trabajos sobre diversidad sexual, o de la población LGBT en general, es importante recalcar que existen diferencias y matices al interior de esa conceptualización, y que de ninguna manera se trata de un conglomerado monolítico de identidades estables. 
identidades aglomeradas bajo las siglas LGBT, es decir, se encuentran combinadas tanto categorías de orientación sexual (lesbianas, gays, bisexuales) junto con identidades de género (transgénero, transexuales), lo cual es importante recalcar debido a que aunque dicho agrupamiento es común en un contexto de activismo o incluso académico, es también impugnado en la práctica y en la teoría (Renn, 2010).

La diversidad sexual como objeto de estudio ha experimentado una serie de cambios; comenzando en el siglo XIX, la sexualidad se ha convertido en un tema donde confluyen debates de orden científico, moral, patológico, religioso y social. La medicina, la psicología, la sexología y la pedagogía eran las disciplinas que se encargaban de abordar el tema de la sexualidad no normativa. (Weeks, 2003). La creciente politización de la sexualidad durante el siglo XX ofreció nuevas posibilidades y nuevas problemáticas, pasando de ser un tema exclusivo de médicos, psiquiatras y religiosos a un asunto analizable desde diferentes perspectivas académicas; la diversidad sexual poco a poco se ha ido convirtiendo en una legítima área de estudios interdisciplinaria (Duggan, 1990).

En Costa Rica, a pesar de que la diversidad sexual es un tema que ha cobrado una creciente relevancia, todavía no existe un documento que sistematice y analice la manera en que se ha desarrollado su estudio a lo largo del tiempo. Se podrían plantear, de manera general, una serie de elementos que ayuden a explicar las razones por las cuales la diversidad sexual como objeto de estudio ha ido en aumento en las últimas tres décadas: la aparición del VIH/SIDA durante la década de 1980 como una problemática inminente de carácter urgente, la despatologización de la homosexualidad por parte de la Organización Mundial de la Salud en 1990, las subsecuentes luchas por despatologizar la transexualidad, la creciente visibilidad y accionar político de grupos sociales y activistas a favor de la diversidad sexual, el apoyo económico de organizaciones de Derechos Humanos para llevar a cabo investigaciones, las renovaciones académicas en varias áreas del conocimiento, la influencia del feminismo y los estudios de género, un mayor apoyo y apertura por parte de instituciones académicas así como la influencia y retroalimentación académica local e internacional y el cambio de un paradigma anclado en la biología a uno que toma como eje central a la sociedad y la cultura.

Sería complicado establecer cuál de esos elementos ha sido el decisivo para explicar la creciente producción académica que ha tenido dicha temática. En una primera instancia es necesario enfatizar que la diversidad sexual es un concepto atravesado por el poder, y por lo tanto, interpela políicamente. En ese sentido, asumir que la única forma, o la forma legítima, de acercarse al tema es bajo una institucionalidad académica universitaria sería muy limitado, y no nos permitiría evidenciar la forma en que la diversidad sexual también ha sido abordada como objeto de estudio por parte de diferentes organizaciones y núcleos de activismo. En este trabajo se toma en cuenta ese amplio umbral de abordajes. 
En el contexto latinoamericano se pueden encontrar importantes esfuerzos por sistematizar la información existente de los estudios sobre la diversidad sexual. Toro-Alfonso (2005) elabora un análisis sobre los trabajos académicos y literarios que abordan la homosexualidad en Latinoamérica, concentrándose en Puerto Rico, evidenciando la gran variedad de temas, así como los distintos enfoques, las metodologías utilizadas y los retos pendientes; al igual que en Costa Rica, los estudios sobre la homosexualidad en Puerto Rico inician en la década de 1980.

Por otro lado, Foster (2008) analiza el estudio de los temas gay en América Latina a partir de la década de los años ochenta del siglo pasado, y para eso divide las investigaciones en seis categorías: las que se centran en discutir sobre definiciones, las que estudian la cuestión de las minorías en relación con las prácticas hegemónicas, las estudios históricos, las que vinculan el género con los estudios culturales, las que relacionan a la masculinidad con la homosexualidad y las que evidencian una intersección entre producción cultural y estudios académico-teóricos. Foster concluye que en los últimos veinticinco años se evidencia una agenda de investigación académica que no puede ser fácilmente ignorada.

Otro aporte importante ha sido el texto coordinado por Brigeiro (2012) acerca de la investigación sobre sexualidad en Colombia y su balance bibliográfico entre 1990 y 2004, es un análisis que abarca muchos temas, entre ellos la diversidad sexual, estableciendo como ejes de análisis la homofobia, la identidad, la socialización, los discursos, la moral, los derechos y la alteridad. López-Vicuña (2004) afirma que hay una reciente efervescencia en los estudios gay y lésbicos latinoamericanos, en donde el creciente número de investigaciones dentro de las Ciencias Sociales parece augurar una mayor complejidad en lugar de una confluencia. El autor hace un balance bibliográfico en tres áreas; primero analiza los estudios literarios del homoerotismo, luego hace una revisión de los textos sobre masculinidad hispana para, finalmente, detallar los documentos que tratan sobre la identidad gay y lésbica en América Latina. Concluye que las investigaciones sobre diversidad sexual seguirán expandiéndose y diversificándose en el futuro.

Por último, Lima (2008) se aproxima a una genealogía del saber queer latinoamericano, prestándoles atención a las principales críticas y debates del feminismo chicano, los estudios gay y lésbicos, y los estudios culturales post marxistas. Este es un balance muy valioso ya que aborda de manera concisa algunas de las más importantes investigaciones influenciadas por la Teoría queer en el contexto latinoamericano y estadounidense.

En el caso de Costa Rica, como ya se mencionó, no existe un documento que aborde el desarrollo de este tipo de estudios. A pesar de que en muchos de los textos nacionales, principalmente, en las tesis universitarias, es posible encontrar estados de la cuestión bastante completos sobre diferentes temáticas 
que se relacionan con la diversidad sexual, estos se hayan dispersos, la mayoría no han sido publicados, y a fin de cuentas no constituyen el punto medular de las investigaciones. Materias con una cierta afinidad a la aquí tratada, como lo es la historia de género (Rodríguez, 2004), los estudios de la mujer (González y Guzmán, 1994) y las masculinidades (Menjívar, 2007) se han visto aproximadas de una forma más sistemática a partir de balances bibliográficos.

En ese sentido, se considera importante este acercamiento exploratorio al desarrollo de los estudios LGBT aplicados al caso de Costa Rica por varias razones: da una mayor visibilización a una temática que sin lugar a dudas ha visto un crecimiento en los últimos años, tiene el potencial de convertirse en una herramienta que facilite la ubicación de textos para futuros investigadores interesados en el tema, posee la capacidad de promover un abordaje más interdisciplinario a partir de los diferentes enfoques y metodologías que existen, ilustra de manera general las materias que se han abordado y su relación con el contexto espacio-temporal, posibilita ubicar lagunas de conocimiento, nuevas problemáticas y formas novedosas de acercarse a los temas, permite contextualizar y comparar el desarrollo académico que ha tenido la diversidad sexual en relación con otras regiones latinoamericanas. Y, finalmente, pone en evidencia el vínculo, directo e indirecto, entre la creación de conocimientos y las políticas nacionales, entre la sexualidad y la política.

\section{Metodología}

Este artículo es un balance realizado por medio de revisión bibliográfica y cuantificación de documentos, se busca crear una base de datos que permita ilustrar y analizar las características de los textos con temática LGBT aplicados al caso de Costa Rica. La búsqueda de textos se hizo de manera tanto física como digitalmente, se exploraron las bases de datos de las bibliotecas nacionales (Universidad de Costa Rica, Universidad Nacional, Instituto Tecnológico de Costa Rica, Universidad Estatal a Distancia, Biblioteca Nacional), las revistas académicas nacionales, las bases de datos internacionales de JSTOR, HAPI, Latindex y Oxford Journals, y también se revisaron los catálogos de librerías nacionales, así como las páginas de internet de diferentes organizaciones activistas y de derechos humanos. El criterio principal de inclusión es que se aborde el tema de la diversidad sexual en Costa Rica como eje central de análisis.

La información fue sistematizada en una base de datos en donde queda consignada la información básica de cada texto. Para llevar a cabo el procesamiento de la información recolectada se creó una serie de categorías: la de autor permite establecer el número de autores por cada texto y la cantidad de textos que cada uno ha escrito, la categoría de género da la posibilidad de contemplar cuántos textos han sido escritos por hombres, mujeres o un equipo mixto de trabajo. La categoría de tipo proporciona una visión detallada del formato de cada uno de los textos, en caso de que sean revistas o libros 
queda consignada la editorial, el número y el volumen, en el caso de las tesis universitarias se establece el grado y la institución a la que pertenece. Para concluir, están las categorías de lugar de publicación, el periodo cubierto y el espacio geográfico analizado, esto con el objetivo de establecer de manera clara el contexto espacio-temporal de cada documento. Los resultados del procesamiento de la información se muestran a través de gráficos y cuadros.

El artículo está organizado de la siguiente manera; primero se establecen cuáles son los tipos de documentos que se logró recolectar, luego se expone la manera en que los textos se han distribuido a lo largo del tiempo y el espacio, seguidamente, se hace un perfil de los autores para luego pasar a la caracterización de las tesis universitarias. Por último, se hace un recuento de cuáles han sido los principales temas de investigación y se concluye con algunas observaciones.

\section{Tipo de documentos}

Después de hacer efectiva la búsqueda de textos, se llegaron a recolectar 130 investigaciones que se ajustan con el criterio de selección antes expuesto. Como primer eje de análisis, es importante establecer qué tipo de documentos se han hecho sobre la temática LGBT aplicados al caso de Costa Rica, por lo que se procede a caracterizarlos en diferentes categorías. El Cuadro 1 expone los tipos de texto encontrados.

\begin{tabular}{|l|c|c|}
\hline $\begin{array}{l}\text { CUADRO 1. DISTRIBUCIÓN EN NÚMEROS ENTEROS Y PORCENTUALES DE 130 TEXTOS DE TEMÁTICA } \\
\text { LGBT APLICADOS AL CASO DE COSTA RICA SEGÚN TIPO DE DOCUMENTO }\end{array}$ \\
\hline \multicolumn{1}{|c|}{ Tipo de documento } & Número & Porcentaje \\
\hline Artículo de libro & 4 & $3 \%$ \\
\hline Artículo de revista & 27 & $21 \%$ \\
\hline Libro & 16 & $12 \%$ \\
\hline Tesis de doctorado & 1 & $1 \%$ \\
\hline Tesis de maestría & 9 & $7 \%$ \\
\hline Tesis de licenciatura & 42 & $32 \%$ \\
\hline Tesis de bachillerato & 2 & $2 \%$ \\
\hline Otros & 29 & $22 \%$ \\
\hline Total & 130 & $100 \%$ \\
\hline Fuente: Elaboración propia. Base de datos sobre textos LGBT aplicados al caso de Costa Rica, 1980-2013. \\
\hline
\end{tabular}


Se evidencia que existe una gran variedad en cuanto a los formatos de presentación de las investigaciones, cada uno de ellos con sus características específicas. Los artículos de libro, los artículos de revista y los libros son documentos que tienen una circulación más amplia debido a que han sido publicados y se pueden acceder de forma directa, juntos conforman el 36\% de las investigaciones recolectadas. El cuadro muestra el considerable aporte que se hace desde los trabajos finales de graduación, siendo las tesis de licenciatura el rubro más importante cuantitativamente. El grupo de tesis constituye el tipo de documento más grande, con un $42 \%$. La categoría de Otros corresponde a documentos como informes digitales, sondeos, ponencias, estudios no publicados y reportes. Es importante tomar en cuenta que en el rubro de Otros hay varios textos publicados por Organizaciones de Derechos Humanos y activistas.

\section{Distribución temporal y espacial}

Uno de los objetivos de este análisis panorámico es evidenciar la manera en que el número de investigaciones sobre la temática estudiada ha ido creciendo durante las últimas tres décadas. Hay que tomar en cuenta que el primer estudio que se encontró, sobre el estudio clínico comparativo entre individuos homosexuales y heterosexuales a través de tests proyectivos gráficos (González, 1980), data de 1980, por lo tanto, para ilustrar de forma clara la dinámica cuantitativamente creciente de los textos, se decidió dividir el periodo comprendido entre 1980-2013 en grupos de cinco años cada uno, a excepción del último periodo que solo comprende cuatro años (2010-2013). El Cuadro 2 sistematiza la información correspondiente, en dos de los textos no se logró determinar su fecha de publicación.

\begin{tabular}{|c|c|}
\hline $\begin{array}{c}\text { CUADRO 2. DISTRIBUCIÓN EN QUINQUENIOS DE } 128 \text { TEXTOS DE TEMÁTICA LGBT APLICA- } \\
\text { DOS AL CASO DE COSA RICA SEGÚN FECHA DE PUBLICACIÓN, 1980-2013 }\end{array}$ \\
\hline Periodo & Número de textos \\
\hline $1980-1984$ & 1 \\
\hline $1985-1989$ & 8 \\
\hline $1990-1994$ & 11 \\
\hline $1995-1999$ & 21 \\
\hline $2000-2004$ & 22 \\
\hline $2005-2009$ & 31 \\
\hline $2010-2013$ & 34 \\
\hline \multicolumn{2}{|c|}{ Fuente: Elaboración propia. Base de datos sobre textos LGBT aplicados al caso de Costa Rica, } \\
1980-2013.
\end{tabular}


Queda claro que el tema de la diversidad sexual ha ido captando de manera creciente la atención de investigadores. El contexto nacional es un elemento que puede dar luces para comprender esta dinámica desde diferentes ángulos. Aunque, tal y como se dijo, el primer texto data de 1980, se podría decir que los estudios académicos empiezan a ser más recurrentes a finales de dicha década debido, principalmente, al surgimiento y recrudecimiento de la epidemia del VIH/SIDA, por lo que muchos de estos trabajos tienen como eje de análisis a la enfermedad y su relación con los grupos de riesgo, entre ellos los homosexuales (Mata, 1988). En el Gráfico 1 se muestra de manera más clara la dinámica de producción creciente de investigaciones sobre el tema:

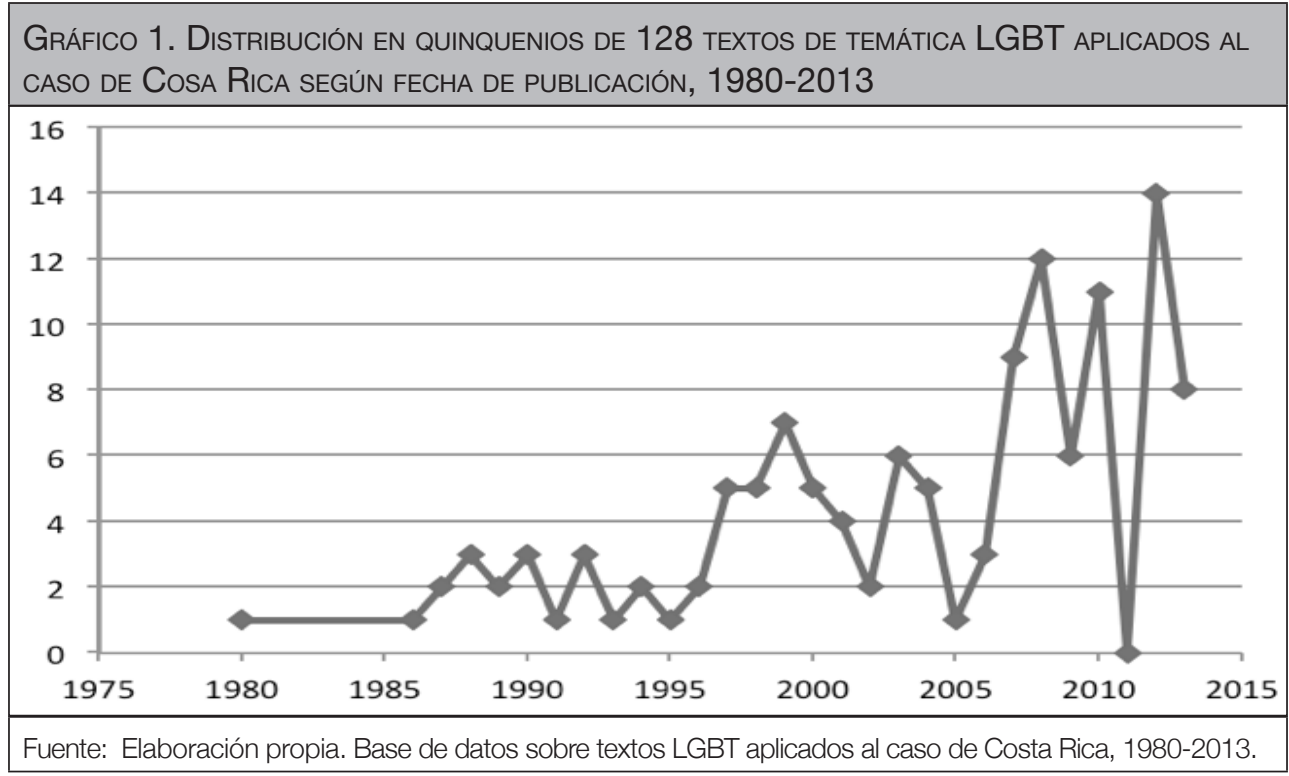

Es interesante ver cómo entre 1990 y 1999 el número de investigaciones casi se duplica. Durante esa década la visibilización de la diversidad sexual en el ámbito público empieza a crecer. Puntos importantes que de una u otra forma influyen en el desarrollo de esta temática fueron: el II Encuentro Lésbico Feminista de América Latina y el Caribe llevado a cabo en Costa Rica en 1990, el I Foro de Diversidad Sexual en Costa Rica en 1998 (Gamboa, 2009), la inscripción formal en el Registro Público de Triángulo Rosa como la primera asociación conformada, explícitamente, por gays y lesbianas (Ramírez y Vargas, 2007) la promulgación de la ley General de VIH/SIDA en 1998 y la despenalización de la sodomía en el Código Penal en 1999 (Murillo, 2003).

Una vez que inicia el siglo XX el movimiento por la diversidad sexual en Costa Rica se ve influenciado por nuevas agendas ${ }^{3}$, entre ellas los derechos 
civiles, patrimoniales y la legislación sobre las parejas del mismo sexo, tema que adquiere una gran importancia gracias a factores como los recursos de amparo frente a la Sala Constitucional, los diferentes proyectos de ley, el activismo con presencia mediática, y las discusiones sobre la posibilidad de convocar a un referéndum con el fin de conocer, en el ámbito nacional, la opinión que tienen los costarricenses frente al tema de las uniones civiles no heterosexuales (Sandoval, 2009). En ese sentido, a partir del siglo XX el tema de la diversidad sexual adquiere una visibilización inédita en el país, lo cual sin lugar a dudas influye en las investigaciones que se llevan a cabo desde la academia.

Otro aspecto importante para el desarrollo de las investigaciones analizadas es el lugar de publicación, la oportunidad de hacer estudios desde instituciones internacionales, ya sean universidades u organizaciones de Derechos Humanos, son una evidencia del dinamismo que tiene la temática LGBT en el mundo y la proyección que tiene Costa Rica sobre esa dinámica (véase Gráfico 2).

\section{Gráfico 2. Distribución de 130 textos de temÁtica LGBT aplicados al CASO dE} Costa Rica según LUGAR de PUblicACIÓN, 1980-2013

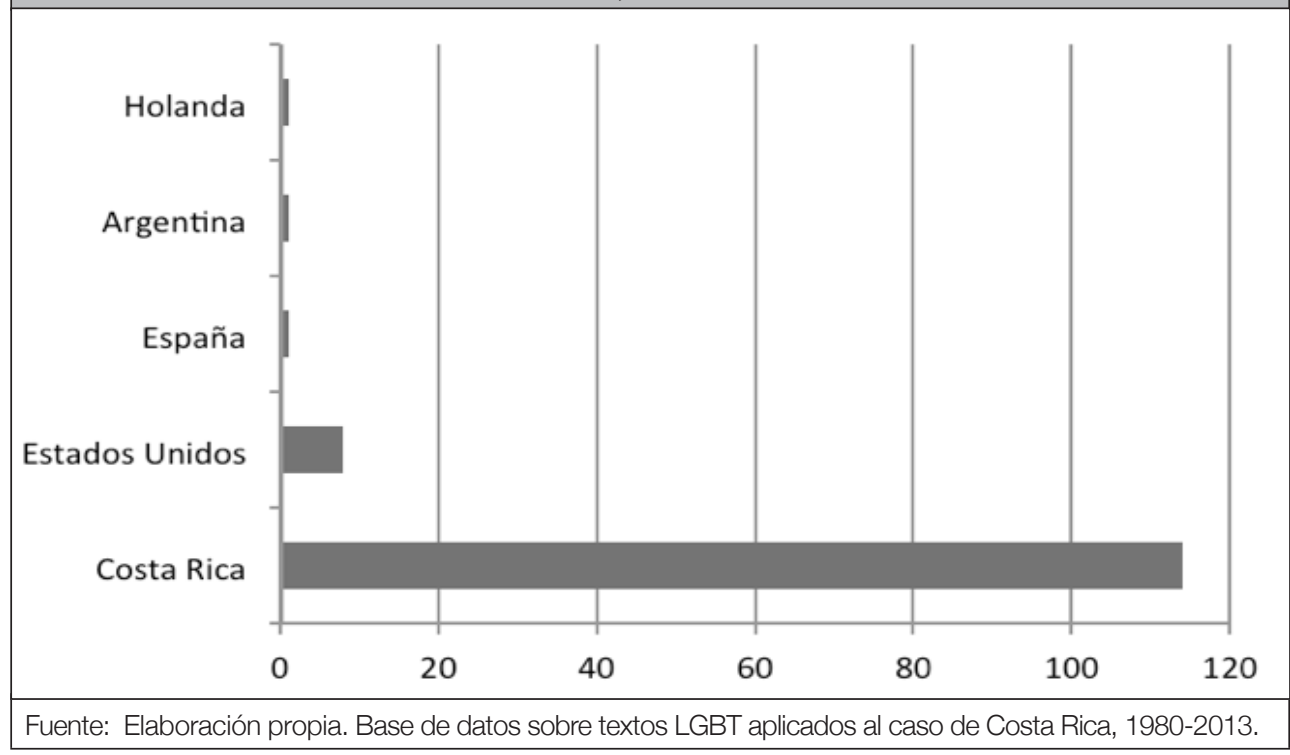

En el Gráfico 2 es posible observar que, efectivamente, Costa Rica sigue siendo el lugar en donde se publican más textos sobre la temática analizada en el contexto costarricense, mientras que otros lugares no representan una

\footnotetext{
A lo largo de la década de 1990, las organizaciones se multiplican y se transforman, algunas cambian de nombre y otras se disuelven de manera intermitente. A inicios del 2000 hubo un primer esfuerzo en Costa Rica por parte de activistas y representantes de la diversidad sexual, para conformar una agenda política en común; sin embargo no sería hasta el año 2010 cuando esta agenda política toma un nuevo impulso con la fundación de la Coalición Nacional de Organizaciones de la Diversidad Sexual, la cual reúne a algunas de la principales organizaciones sobre esta temática en el país, su agenda política se puede revisar en línea (www.conodis.org).
} 
proporción grande. El texto de Holanda corresponde a una tesis de maestría en sociología de la Universidad de Ámsterdam (Álvarez, 2012), el texto argentino es la publicación en libro por parte de la Editorial Elaleph (Vargas, 2003) de lo que, originalmente, fue una tesis de maestría en Antropología de la Universidad de Costa Rica (Vargas, 2001), la investigación publicada en España es un artículo en Nómadas: Revista crítica de ciencias sociales y jurídicas (Gamboa, 2008), mientras que los documentos publicados en Estados Unidos son nueve, la mayoría de los cuales son artículos de revista.

La construcción de alianzas con entes internacionales ha sido un factor muy importante para el desarrollo de muchas investigaciones, el aporte financiero de grupos como el Instituto Humanista de Cooperación y Desarrollo (HIVOS), COC Nederland, y otras organizaciones de lucha contra el VIH/SIDA posibilitaron la publicación de varios libros por parte del Instituto Latinoamericano de Prevención y Educación en Salud (ILPES); de los dieciséis libros publicados durante el periodo analizado, diez fueron fruto de esta editorial costarricense. El Centro de Investigación y Promoción para América Central de Derechos Humanos (CIPAC) también ha sido una organización bastante prolíica, en parte gracias al financiamiento externo, 21 de los textos corresponden a esta entidad que desde la década de 1990 hasta la actualidad ha publicado investigaciones de diferentes temáticas relacionadas con la diversidad sexual y los Derechos Humanos. Es una tarea pendiente el proyectar las investigaciones costarricenses hacia el exterior, mediante vínculos institucionales, reseñas académicas, indexación en bases de datos internacionales, y lo más importante, mantener una visión crítica y actualizada sobre las disputas teóricas y epistemológicas que atraviesan el estudio de la sexualidad. Todos estos elementos pueden contribuir a que el tema de la diversidad sexual aplicado al caso de Costa Rica sea conocido no solo a nivel nacional.

\section{Perfil de los autores}

Además de analizar aspectos medulares de las investigaciones, es importante tener una noción acerca de quiénes son los autores. La composición según género puede dar luces acerca del interés y apertura que han tenido tanto hombres como mujeres hacia la temática de la diversidad sexual; aquí se hace referencia a la caracterización binaria como forma de facilitar el análisis, y esto es necesario enfatizarlo porque, por la misma naturaleza de los documentos, existe una crítica hacia esa visión estable e inmutable del sexo/género como una realidad biológicamente determinada. El Cuadro 3 muestra la composición por género de los autores.

Es interesante que en términos generales exista un equilibrio entre los textos llevados a cabo por mujeres y por hombres, aunque no es un objetivo de este análisis ahondar en detalles sobre la orientación sexual o la identidad de género de las y los autores, sería revelador en un futuro establecer hasta qué punto el 
interés por la temática de la diversidad sexual desde una óptica académica y activista está atravesada por cuestiones personales. Se rescata también el contingente de textos que fueron hechos por un equipo mixto de trabajo.

\begin{tabular}{|c|c|c|}
\hline \multicolumn{3}{|c|}{ 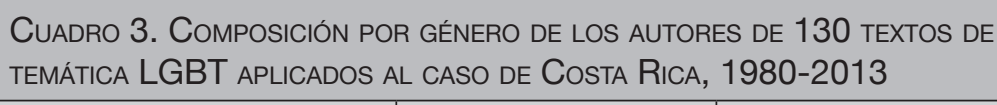 } \\
\hline Género & Número & Porcentaje \\
\hline Hombre & 57 & $44 \%$ \\
\hline Mujer & 56 & $43 \%$ \\
\hline Mixto & 17 & $13 \%$ \\
\hline
\end{tabular}

\section{Caracterización de las tesis}

Las tesis son un tipo de texto que, al contrario de libros y revistas, se encuentran en repositorios con un acceso más limitado, usualmente una biblioteca universitaria. Representan el $42 \%$ del total de textos recolectados para la base de datos, por lo que es importante abordarlas de manera detallada. Las diferentes tesis son una evidencia del interés que, dentro de los mismos programas de educación superior, existe sobre la diversidad sexual, tema que ha estado más abierto en ciertas Escuelas y que ha promovido la investigación de problemáticas bastante novedosas en el contexto nacional. Al hacer la búsqueda, se encontró que se han producido tesis en cinco instituciones universitarias: cuarenta y seis en la Universidad de Costa Rica, seis en la Universidad Nacional de Costa Rica, una en la Universidad de Ámsterdam, dos en la Universidad Internacional de las Américas y una en la Universidad Veritas. En el Cuadro 4 se puede vislumbrar a qué escuelas pertenecen las diferentes tesis recolectadas.

Es notable el predominio que tiene la Universidad de Costa Rica en la producción de trabajos finales de graduación de grado y posgrado con respecto a la temática de la diversidad sexual, como ya se señaló anteriormente, la mayoría de estas tesis son de licenciatura. A pesar de existir una gran variedad de escuelas y departamentos cuya estructura permite el abordaje de objetos de estudio como los que aquí se analizan, es clara la preponderancia de esfuerzos investigativos por parte de la Escuela de Psicología, seguida por la Facultad de Derecho y la Escuela de Trabajo Social. El hecho de que exista la obligación de colegiarse en ciertas carreras posiblemente influye en la cantidad de tesis de licenciatura que se realizan. Además, puede que dentro de la misma dinámica de las Escuelas exista una mayor apertura hacia la exploración de temas que, a pesar de todo, a nivel nacional siguen siendo relativamente invisibilizados. 


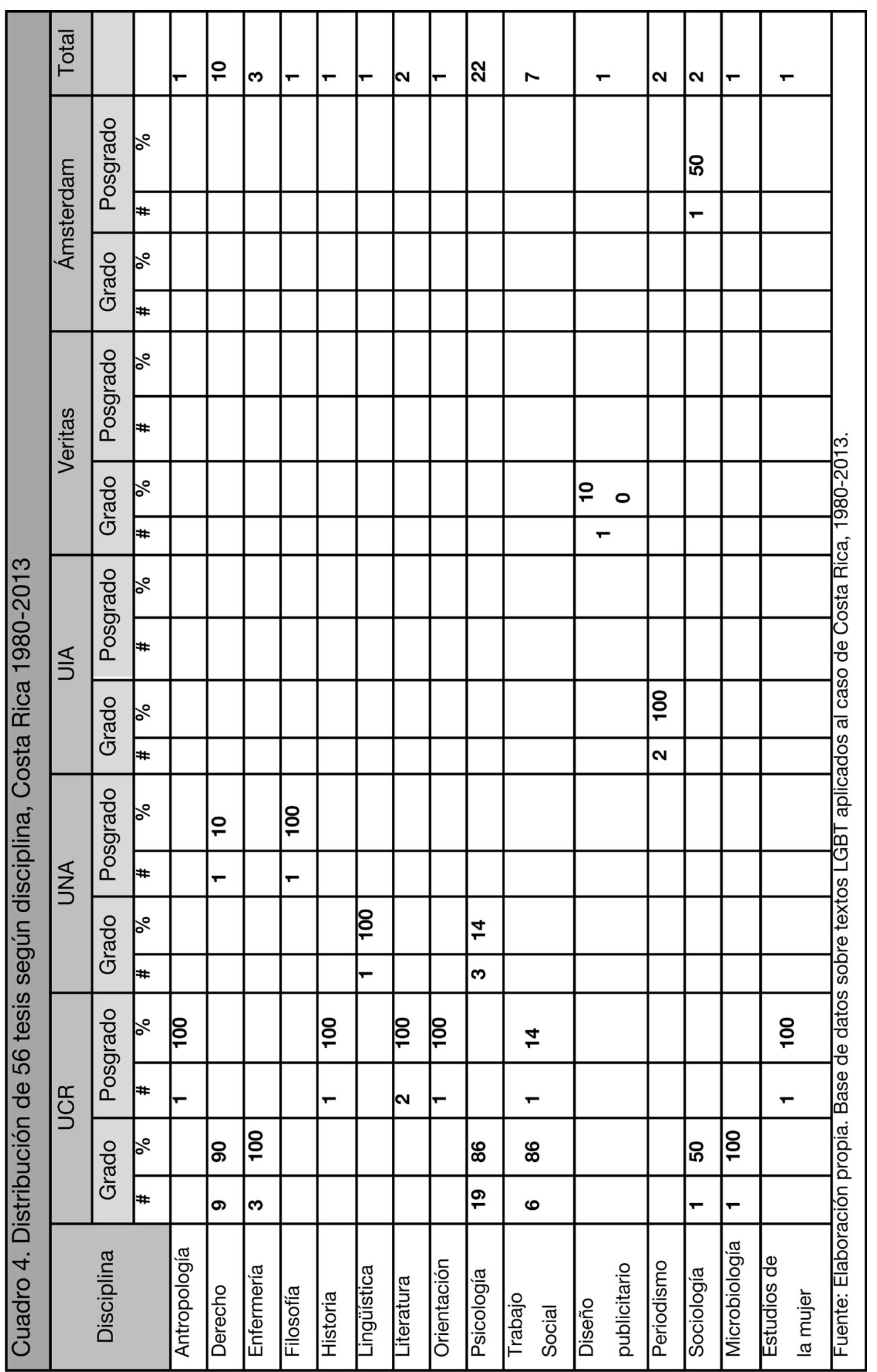




\section{Temáticas en construcción}

Uno de los objetivos principales de esta investigación es establecer cuáles han sido los temas abordados en los textos recolectados. Los primeros documentos enfocados en Costa Rica que trataron el tema de la diversidad sexual tuvieron como principal eje de análisis al VIH/SIDA, en ese sentido muchos de estos primeros esfuerzos se vieron teñidos por una perspectiva médica, incluso biologicista, no es de extrañar que muchos de los primeros artículos fueran publicados por la Revista Costarricense de Ciencias Médicas; en ese sentido se encuentran temas como la relación entre comportamientos riesgosos y el consumo de drogas (Rodríguez, 1986; Mata y Ramírez, 1987; Mata, Ramírez y Rosero, 1987) las conductas sexuales y las proyecciones en cuanto a que tan grande podía llegar a ser la epidemia (Mata, 1988). En los últimos años de la década de 1980 también surge otro tipo de cuestiones, como por ejemplo, el diagnóstico de pseudohermafroditismo (Chacón y Lara, 1987) el cual es un tema que, a pesar de su aparición temprana, ha sido escasamente desarrollado ${ }^{4}$. Otro eje investigativo que nace en esa misma década es el de la transexualidad, específicamente la práctica de emasculación $n^{5}$, y su relación con el Derecho Penal costarricense (Guillén, 1989). Finalmente, en la década de 1980 salió a la luz uno de los libros más importantes desde una perspectiva histórica en cuanto a la diversidad sexual: La formación de una contracultura. Homosexualismo y SIDA en Costa Rica de Schifter (1989).

$4 \quad$ En este caso se podría tomar a este texto como un estudio pionero sobre la intersexualidad, la cual en términos estrictamente médicos es explicada como una anomalía congénita del sistema reproductivo y sexual, en otras palabras, las personas intersexuales son aquellas que nacen con condiciones físicas que resultan en anomalías reproductivas o anomalías cromosómicas atípicas (Koyama y Weasel, 2002). No obstante, este discurso médico ha sido desafiado por críticas feministas las cuales problematizan el binario biológico del sexo, argumentando por una fluidez del género, y localizando nuevas intersecciones entre sexo y género. Las relaciones de poder que establecen guías normativas para el comportamiento de las personas acorde a su género también incluye guías para normalizar el aspecto material del cuerpo. El caso de las personas intersexuales ejemplifica esta dinámica ya que sus cuerpos solo pueden existir socialmente si se ajustan a los esquemas de cómo debe verse y actuar un hombre o una mujer, aunado a las intervenciones médicas que buscan, mediante cirugías o procesos hormonales, fijar el cuerpo intersexual en uno u otro sexo/género, pero nunca en el medio (Turner, 1999). En ese sentido, la intersexualidad es un concepto que problematiza las visiones esencialistas de la sexualidad, en el caso costarricense muchas veces se agrega la sigla "L" a "LGBTI" como forma de reivindicar, desde el activismo, la mayor cantidad de identidades posibles, no obstante para este trabajo se optó por no hacerlo ya que desde la producción académica no ha sido un tema lo suficientemente abordado y su presencia en las investigaciones es casi nula. Es un desafío aproximarse a esta temática tan poco explorada, además de que es un imperativo debido a que "Ios individuos intersexuales, al no coincidir en la clasificación masculina o femenina, no están incorporados en las políticas y leyes gubernamentales de la mayoría de países y Costa Rica no es la excepción" (Vargas, 2013, 150). Para una visión más profunda de una experiencia intersexual particular en Costa Rica se puede leer la entrevista hecha a Natasha Jiménez en el libro Interdicciones (Cabral, 2009, 123-130).

5 Por emasculación se entiende el procedimiento quirúrgico que se aplica a las personas con el fin de extirpar los órganos genitales masculinos externos (pene y testículos). Actualmente, se le conoce por el término de cirugía de reasignación genital/sexual. 
Durante la última década del siglo XX hay una abundancia de publicaciones por parte del ILPES, se publica la primera Encuesta Nacional sobre SIDA (Schifter y Madrigal, 1990), y a lo largo de esa década dicha enfermedad se convierte en uno de los motores para la investigación sobre la diversidad sexual (Hidalgo y Flores, 1990; Gutiérrez, 1992, 1994, 1995; Madrigal, 1998; Muñoz, 1998; Güell, 1999), se llevan a cabo investigaciones sobre salud pública, uso de condones, se siguen dando estudios sobre la legislación costarricense frente a la transexualidad (Alvarado y Soto, 1993), surge uno de los primeros artículos hechos en el exterior enfocado en Costa Rica como lo es el estudio de Thayer (1997) publicado en la revista Social Problems, el cual aborda el tema del activismo lésbico a partir de una perspectiva comparativa entre Costa Rica y Nicaragua. El CIPAC inicia sus investigaciones adentrándose en el tema de la discriminación laboral por orientación sexual, se dan estudios sobre la comunidad bisexual (Schifter, Madrigal y Aggleton, 1996). Y, por último, en el año 1999 entra en escena un tema que eventualmente se convertirá en uno de los catalizadores de la investigación y el activismo: las parejas del mismo sexo. Los primeros esfuerzos se concentran en las historias de vida, las vivencias entre parejas de mujeres autodefinidas como lesbianas (Chinchilla y Jones, 1999).

Durante el primer lustro del siglo XXI, se abordan temas como la prostitución (Gamboa, 2000), el suicidio (Suárez y Madrigal, 2000), el travestismo (Madrigal y Gallo, 2000), el VIH/SIDA continúa siendo un eje de análisis importante, empiezan a surgir los estudios sobre literatura homoerótica en Costa Rica (Vega, 2000; Carrasco, 2003). Se continua explorando el tema de la transexualidad (Pérez, 2001). El lesbianismo y las experiencias de vida de las mujeres empieza a tomar fuerza como objeto de estudio (Suárez, 2000; Serrano, 2002; Aguilar, 2004, Marín, 2004). Sin embargo, el tema que monopoliza la mayoría de las investigaciones es el de la figura de la pareja del mismo sexo y su relación con la legislación costarricense y los derechos civiles. En ese sentido, hay esfuerzos importantes desde la antropología (Vargas, 2001), la psicología (Alfaro y Cabrera, 2002; Guzmán y Méndez, 2003) y el derecho (Murillo, 2003).

A partir de la segunda mitad de la década del 2000 hay un aumento en el número de textos, como ya se vio antes, este ha sido de los períodos cuantitativamente más importantes en relación con la producción de investigaciones sobre diversidad sexual. El estudio de la producción literaria que aborda aspectos de la sexualidad no normativa sigue estando presente (Obando, 2008; Coto, 2007). El tema de la organización politizada y el activismo adquiere un papel importante (Ramírez y Vargas, 2007; Wilson, 2007; Gamboa, 2008; Trejos, 2008), el desarrollo de los diferentes grupos organizados se convierte en un eje de análisis muy importante.

Surgen estudios novedosos con perspectiva histórica que tratan el tema de la sodomía (Alvarenga, 2007), llevando el estudio de la sexualidad a periodos que usualmente no son tomados en cuenta como la primera mitad del 
siglo XX. Abundan las investigaciones que se concentran en los pronunciamientos institucionales sobre temas de la diversidad sexual y los derechos civiles (Hernández y Valverde, 2007; Soto, 2008; Suárez, 2008; González, 2009; Fournier, 2009) en ese sentido los recursos de amparo, los debates legislativos y la posición de figuras públicas se transforman en ejes de análisis y objetos de estudio importantes. El tema del matrimonio, la unión civil, la unión de hecho y las sociedades de convivencia son un ejemplo de cómo el contexto nacional influye en la producción de investigación académica. La transexualidad sigue siendo un tema que poco a poco va tomando más espacio (Ollé, 2008). Este análisis panorámico da cuenta de cómo algunas identidades han sido más estudiadas que otras, en el caso costarricense la homosexualidad masculina es la preponderante, seguida por el lesbianismo; mientras que la transexualidad, la bisexualidad y la intersexualidad siguen estando menos exploradas.

En los últimos cuatro años, la producción académica ha seguido dando frutos incorporando nuevas perspectivas como, por ejemplo, la lingüística (Marín, 2012). El tema trans continúa siendo analizado desde diferentes perspectivas (Castro y Chaverri, 2011; Días, 2012; Rostrán, 2012; Soto, 2013). Los Derechos Humanos, la organización activista, las historias de vida, los estudios de caso, la lucha por la despatologización, y las legislaciones siguen siendo temas preponderantes, mientras que el VIH/SIDA ha dejado de ser el tema principal, aunque continúa como eje de análisis principalmente desde organizaciones de Derechos Humanos como CIPAC. El Cuadro 5 muestra de manera panorámica y cronológica el desarrollo de estos estudios, sus temáticas principales y los aportes generales.

En cuanto a los espacios temporales de análisis cubiertos por las investigaciones son muy pocas las que analizan el período anterior a la década de 1980, las que sí lo hacen, a menudo, tienen una perspectiva histórica. En este caso hay que tomar en cuenta que la mayoría de las fuentes disponibles datan de años recientes, por lo que continúa siendo un desafío el explorar a la sexualidad no normativa del pasado. La década de los ochenta es tomada por muchos documentos como el punto de inicio para la periodización, el surgimiento del VIH/SIDA marca un límite, una frontera a partir de la cual se empiezan a gestar las problemáticas. Muchos de los estudios al ser situacionales, principalmente, cuando son estudios de caso, se concentran en uno o dos años específicos, mientras que hay otro grupo que abarca periodos medianos de tiempo, por lo general un par de décadas. En lo que respecta al espacio geográfico, el Gráfico 3 ilustra cuáles han sido las tendencias. 


\begin{tabular}{|c|c|c|}
\hline Periodo & Temáticas Principales & Aportes Generales \\
\hline $1980-1989$ & $\begin{array}{l}\text {-VIH/SIDA } \\
\text {-Activismo }\end{array}$ & $\begin{array}{l}\text { Incipiente visibilización. } \\
\text { Estudios pioneros que abren } \\
\text { camino para nuevas } \\
\text { problemáticas. }\end{array}$ \\
\hline 1990-1994 & $\begin{array}{l}\text {-VIH/SIDA } \\
\text {-Homofobia } \\
\text {-Identidades trans }\end{array}$ & $\begin{array}{l}\text { Aproximaciones al tema trans } \\
\text { desde el derecho. } \\
\text { Abordaje de la homofobia como } \\
\text { una problemática social. }\end{array}$ \\
\hline 1995-1999 & $\begin{array}{l}\text {-VIH/SIDA } \\
\text {-Parejas del mismo sexo } \\
\text {-Derechos civiles } \\
\text {-Movimientos políticos }\end{array}$ & $\begin{array}{l}\text { Nuevas perspectivas desde la } \\
\text { psicología, el trabajo social y el } \\
\text { derecho. } \\
\text { Exploración de nuevas } \\
\text { temáticas que vinculan la } \\
\text { sexualidad con la política. }\end{array}$ \\
\hline 2000-2004 & $\begin{array}{l}\text {-Parejas del mismo sexo } \\
\text {-Literatura } \\
\text {-Legislación y política } \\
\text {-Identidades lésbicas }\end{array}$ & $\begin{array}{l}\text { Primeros análisis sobre literatura } \\
\text { homoerótica costarricense. } \\
\text { Abordaje de diferentes } \\
\text { derechos civiles y la necesidad } \\
\text { de legislación. }\end{array}$ \\
\hline 2005-2009 & $\begin{array}{l}\text {-Discursos sobre diversidad } \\
\text { sexual (literatura, periódicos, } \\
\text { expedientes médicos, archivos } \\
\text { judiciales) } \\
\text {-Activismo y organización } \\
\text { política } \\
\text {-Vivencias cotidianas } \\
\text {-Legislación sobre parejas del } \\
\text { mismo sexo }\end{array}$ & $\begin{array}{l}\text { Nuevos análisis desde la histo- } \\
\text { ria, trabajo social, psicología y } \\
\text { derecho. } \\
\text { Abordaje a partir de estudios de } \\
\text { caso e historias de vida. } \\
\text { Exploración de fuentes nove- } \\
\text { dosas. }\end{array}$ \\
\hline 2010-2013 & $\begin{array}{l}\text {-Derechos humanos } \\
\text {-Despatologización } \\
\text {-Salud sexual } \\
\text {-Identidades trans } \\
\text {-Experiencias de vida }\end{array}$ & $\begin{array}{l}\text { Mezcla de trabajos que tienen } \\
\text { una perspectiva coyuntural y de } \\
\text { proceso. } \\
\text { Espacio público como } \\
\text { escenario de análisis. } \\
\text { Balance en torno a los } \\
\text { diferentes avances sobre Dere- } \\
\text { chos Humanos. } \\
\text { Énfasis en la cotidianidad y las } \\
\text { experiencias de vida. }\end{array}$ \\
\hline
\end{tabular}




\section{GRÁFICO 3. DistRIBUCIÓN DE 130 TEXTOS DE TEMÁtICA LGBT APLICADOS AL CASO DE} Costa RICA SEGÚN ESPACIO GEOGRÁFICO ANALIZADO, 1980-2013

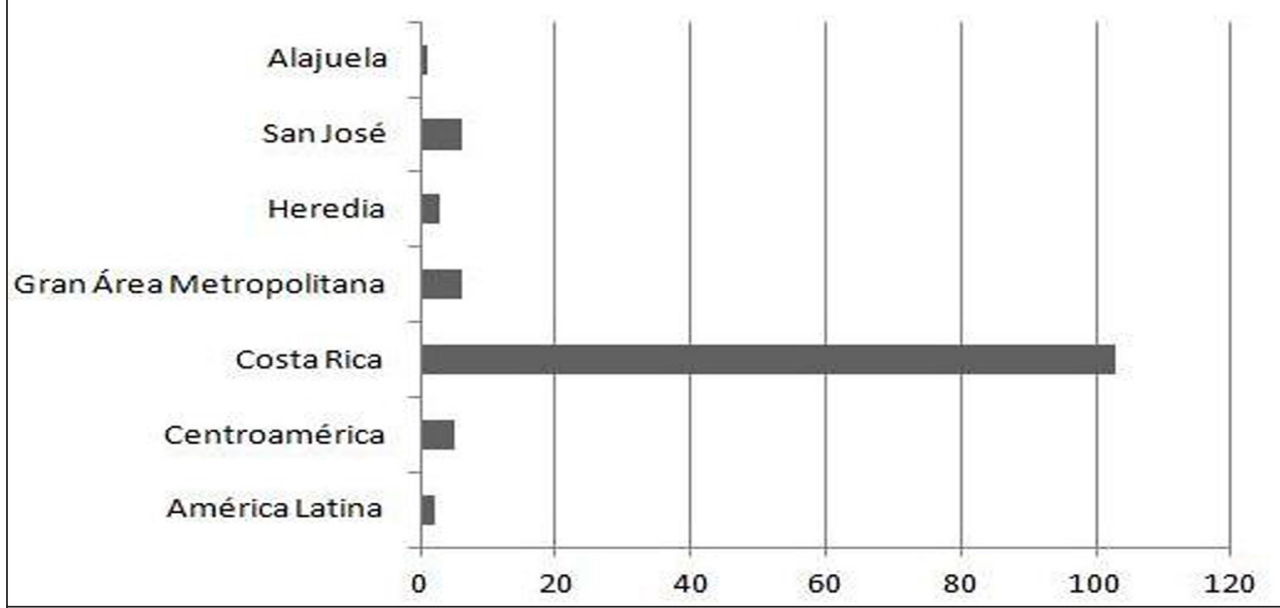

Fuente: Elaboración propia. Base de datos sobre textos LGBT aplicados al caso de Costa Rica, 1980-2013.

A pesar de que algunas de las investigaciones establecen claramente cuál es el espacio geográfico analizado, la mayoría toman al país como un todo. Las que abordan a América Latina o a Centroamérica como espacio principal, usualmente, tocan el tema de Costa Rica de manera parcial. Se pone en evidencia el carácter urbano de la temática, siendo ciudades como Heredia, Alajuela, San José y, la misma Gran Área Metropolitana, los espacios que sí se detallan.

\section{Conclusiones}

El tema de la diversidad sexual entendida como la expresión de una identidad gay, lesbiana, bisexual, transexual o bisexual es un tema que ha adquirido una mayor relevancia en los últimos años, para entender esta dinámica creciente de producción académica hay que tener en cuenta el contexto nacional e internacional; el surgimiento del VIH/SIDA a finales de la década de 1980, la eventual organización politizada en pro de la diversidad sexual, una mayor apertura mediática y política hacia el tema, así como la influencia de agendas mundiales que privilegian el tema de los derechos civiles son todos elementos que hay que considerar al abordar el desarrollo de las investigaciones LGBT aplicadas al caso de Costa Rica.

Se constató que han habido esfuerzos importantes para publicar investigaciones en diferentes formatos, los artículos de revista y las tesis de licenciatura son los principales medios que utilizan los investigadores para dar a conocer sus hallazgos. Además, existe un equilibrio en cuanto al género de los investigadores; la diversidad sexual parece ser un tema que interpela tanto a hombres 
como a mujeres, al mismo tiempo que se han conformado grupos mixtos de investigación. Costa Rica sigue siendo el principal lugar de publicación y la Universidad de Costa Rica es la institución en donde se realiza más estudios sobre el tema, especialmente, la Escuela de Psicología.

Al establecer cuáles han sido las principales temáticas abordadas por los investigadores se puede establecer una serie de vacíos investigativos. La bisexualidad y la intersexualidad siguen siendo temas raramente estudiados. Al mismo tiempo la heterosexualidad como categoría de análisis es inexistente, lo cual deja ver que incluso en la academia algunos temas se dan por sentado o, simplemente, no se problematizan. Es interesante constatar que la transexualidad como objeto de estudio ha estado presente en el interés de los investigadores durante todo el periodo analizado, aunque el número de textos no es tan amplio como los que tratan el tema gay o lesbiano, es importante remarcar que, como categoría de identidad de género, lo trans constituye un elemento analizable desde diferentes perspectivas como la legal, la médica, la social y la psicológica.

Otro vacío se relaciona con el espacio temporal, todavía quedan pendientes las investigaciones de larga duración, donde la prioridad la tengan los procesos y las estructuras sociales amplias. El siglo XIX e incluso el periodo colonial podrían ser nuevos espacios de análisis en donde, mediante la utilización de fuentes novedosas y metodologías pertinentes, se pueda ahondar en los largos procesos de construcción de las sexualidades costarricenses. De igual forma, los análisis comparativos son otro vacío que se evidenció al hacer la revisión bibliográfica, la influencia de otros contextos son claves para entender la manera en que la diversidad sexual, y, principalmente, su legislación y activismo, se va posicionando en el discurso público; en ese sentido, comparar la experiencia de Costa Rica con otros países latinoamericanos, e incluso con espacios anglosajones, permitiría expandir el grado de análisis, y evidenciaría las conexiones y las redes que existen en la región.

En esta visión panorámica de las temáticas se puede afirmar que, aunque la teoría queer posiblemente ha impregnado la posición teórica de algunas de las investigaciones, todavía queda pendiente enfatizar el enlace de la diversidad sexual, y la sexualidad en general, con aspectos de orden económico, racial, educativo, entre otros. La sexualidad es una construcción social que se ve atravesada por una serie de elementos y dispositivos de poder, y esa dinámica tiene un gran potencial para ser investigada de manera más sistemática en un futuro. El papel de las universidades para el desarrollo de estas temáticas es crucial:

Frente a sociedades con crisis económicas y políticas crónicas, por no decir permanentes, la supervivencia a corto plazo y los problemas de gobernabilidad absorben las energías sociales y políticas, que deberían invertirse en ese trabajo lento, cotidiano, a mediano y largo plazo, que es el enfrentar con éxito las desigualdades, las discriminaciones, los conflictos y las necesidades de participación de los ciudadanos[...]. Las universidades han dado cuenta de 
este fenómeno y se abren poco a poco a los estudios sobre minorías sexuales, en el entendido de que la lucha contra la discriminación es requisito esencial de la convivencia en sociedades donde se relajan los lazos tradicionales y es preciso atender a problemas muy complejos de identidad y organización social y política. En este sentido, las universidades latinoamericanas están saliendo del clóset (Kozak, 2006, 136-137).

Cabe preguntarse ¿hasta qué punto se han salido del clóset las universidades costarricenses? Si nos enfocamos exclusivamente en el carácter cuantitativo, se podría afirmar que han habido esfuerzos importantes desde diferentes Escuelas para abordar esta temática, "en las universidades públicas la investigación relacionada con las comunidades no heterosexuales experimentó un crecimiento significativo desde finales del siglo XXI" (Molina, $2013,18)$. Sin embargo, es necesario enfatizar que la diversidad sexual como objeto de estudio es diferente a la diversidad sexual como experiencia, como vivencia cotidiana.

Esa diferencia ha sido entendida por las tres universidades estatales del país, pues en el 2008 se firma un decreto por parte del gobierno para celebrar anualmente el día contra la homofobia cada 17 de mayo (Decreto Ejecutivo, 2008) a raíz de esto, un año después en el 2009 se lleva a cabo el primer Festival Interuniversitario, como una manera de conmemorar ese día (Mata, 2009). En ese sentido, la diversidad sexual deja de ser un concepto abstracto, académico, para convertirse en una reivindicación, en una puesta en escena política, en una manifestación material de los avances y limitaciones que enfrentan muchas personas en su cotidianidad. Finalmente, también es importante cuestionar hasta qué punto hay una efectiva circulación, acceso, publicación y divulgación del conocimiento. La sexualidad tiene un trasfondo político, por lo que la inquietud académica no debe quedarse aislada de las discusiones nacionales e internacionales sobre la diversidad sexual, sería importante establecer un puente entre la producción académica y las organizaciones de Derechos Humanos y activistas.

\section{Referencias}

Aguilar Sandi Evelyn Priscila. (2004). Las uniones de hecho, su legislación y la violación de los derechos humanos de las lesbianas. Tesis de maestría en Derechos Humanos y Educación para la Paz, Universidad Nacional, Heredia, Costa Rica.

Alfaro Soto, Jeffry y Cabrera Ramírez, Mónica. (2002). Hacer el amor entre nombres: la construcción y el desarrollo del vínculo amoroso en la homosexualidad masculina. Un análisis psicosocial. Tesis de licenciatura en Psicología, Universidad de Costa Rica, San José, Costa Rica. 
Alvarado Castro, Douglas y Soto Suarez, Juan. (1993). La readaptación sexoorgánica en los transexuales y sus efectos en el ordenamiento jurídico costarricense. Tesis de licenciatura en Derecho, Universidad de Costa Rica, San José, Costa Rica.

Alvarenga Venutolo, Patricia. (2007). ¿Sodomitas u homosexuales? El homoerotismo y el sistema penal costarricense en la primera mitad del siglo XX. Revista Página Literal, Extraña Intimidad, 7(1), 48-61.

Álvarez Hernández, Carlos Andrés. (2012). Fearing the loca Gay men in public spaces in Costa Rica. Tesis de maestría en Sociología, Universidad de Ámsterdam, Holanda.

Brigeiro, Mauro. (2010). La investigación sobre sexualidad en Colombia (19902004): balance bibliográfico. Bogotá: Universidad Nacional de Colombia. Facultad de Ciencias Humanas. Escuela de Género.

Cabral, Mauro. (2009). Interdicciones escrituras sobre la intersexualidad. Córdoba: Editorial Anarrés.

Carrasco, Candide. (2003). Voces gay en la narrativa costarricense. Letras, 1(15), 81-101.

Castro Villalobos Cynthia y Chaverri Sánchez, Melania. (2011). Estudios de casos de personas trans (transexuales, transgéneros y travestis): experiencias vividas en el espacio laboral de Costa Rica. Tesis de licenciatura en Psicología, Universidad de Costa Rica, San José, Costa Rica.

Chacón Echeverría, Laura y Lara Guardia, Doris. (1987). La construcción de la identidad sexual en cinco adolescentes diagnosticados pseudohermafroditas. Tesis de licenciatura en Psicología, Universidad de Costa Rica, San José, Costa Rica.

Chinchilla Arce, Katia y Jones Solórzano, Patricia. (1999). Análisis de las vivencias y concepciones acerca de las relaciones de pareja de un grupo de mujeres adultas autodefinidas como lesbianas: una experiencia de grupo focal. Tesis de licenciatura en Psicología, Universidad de Costa Rica, San José, Costa Rica.

Coto Rivel, Sergio Andrés. (2007). Espacios de marginalidad y nuevas propuestas de género: La construcción del discurso homoerótico en la novela Tumbas con paisajes pintados en rosa de José Ricardo Chávez. Tesis de maestría en Literatura, Universidad de Costa Rica, San José, Costa Rica.

Decreto Ejecutivo No. 34399-S. (2008, 25 de marzo). Declaratoria oficial del día contra la homofobia, La Gaceta, No. 58. 
Días Pasten, Soledad. (2012). La situación en derechos y salud sexual y reproductiva de personas lesbianas, trans e intersex en Costa Rica. Costa Rica: Mulabi.

Dugan, Lissa. (1990). From Instincts to Politics: Writing the History of Sexuality in the U.S. The Journal of Sex Research, 27(1), 95-109. Recuperado de la base de datos Jstor.

Foster, David William. (2008). El estudio de los temas gay em América Latina desde 1980. Revista Iberoamericana, 74(225), 923-947.

Fournier, Marco Vinicio. (2009). Actitudes y estereotipos del personal de Ios EBAIS hacia la diversidad sexual en el Valle Central de Costa Rica. Costa Rica: Centro de Investigación y Promoción para América Central de Derechos Humanos.

Gamboa Barboza, Isabel. (2000). Los burdeles de la isla: relaciones eróticoafectivas entre mujeres. Ciencias Sociales, 88, 77-85.

Gamboa Barboza, Isabel. (2008). Sociabilidad e identidad en el campo sexual en Costa Rica, 1980-2004. Nómadas Revista Crítica de Ciencias Sociales y Jurídicas, 18(2), 1-12.

Gamboa Barboza, Isabel. (2009). En el Hospital Psiquiátrico. El sexo como locura. San José: Grafos Litografía.

González Agüero, Saray. (1980). Estudio clínico comparativo entre individuos homosexuales y heterosexuales a través de tests proyectivos gráficos: la figura humana y el dibujo de la familia. Tesis de licenciatura en Psicología, Universidad de Costa Rica, San José, Costa Rica.

González Suárez, Mirta. (2009). Proyecto de ley de unión civil entre personas del mismo sexo: Posiciones a favor y en contra. Revista Wímb lu, 4(1), 11-30.

González Suárez, Mirta y Guzmán Stein, Laura. (1994). Los estudios de la mujer en Costa Rica: Desafiando el pasado, construyendo el futuro. Ciencias Sociales, 65, 7-16.

Guillén Rodríguez, lleana. (1989). La emasculación en transexuales, su regulación en el derecho penal costarricense. Tesis de licenciatura en Derecho, Universidad de Costa Rica, San José, Costa Rica.

Güell Durán, Ana Josefina. (1999). Experiencia terapéutica grupal para personas VIH/SIDA homosexuales: un aporte desde la perspectiva cognoscitiva. Tesis de maestría en Trabajo Social, Universidad de Costa Rica, San José, Costa Rica.

Gutiérrez Doña, Benicio. (1992). SIDA y homosexualismo: dimensiones de la personalidad, la historia vital y medioambientales vinculadas al SIDA, al 
estrés y a los procesos cognoscitivos en hombres homosexuales del área metropolitana. Tesis de licenciatura en Psicología, Universidad de Costa Rica, San José, Costa Rica.

Gutiérrez Doña, Benicio. (1994). Estrategias de afrontamiento al SIDA y dimensiones de la personalidad. Ciencias Sociales, 66, 93-105.

Gutiérrez Doña, Benicio. (1995). Riesgo percibido de contraer SIDA: Comportamiento sexual y cambios en el comportamiento en hombres homosexuales. Ciencias Sociales, 69, 107-112.

Guzmán Delgado, Allan y Méndez Montero, Miriam. (2003). Pareja homosexual masculina: representación social y dinámica del vínculo de pareja. Tesis de licenciatura en Psicología, Universidad de Costa Rica, San José, Costa Rica.

Hernández Leiva, Mónica y Valverde Phillips, Carmen Laura. (2007). Estudio sobre la inconstitucionalidad de la prohibición del matrimonio entre personas del mismo sexo. Tesis de licenciatura en Derecho, Universidad de Costa Rica, San José, Costa Rica.

Hidalgo, Roxana y Flores González, Mercedes. (1990). Aspectos psicosociales de la potencialidad hacia el autoritarismo, moral sexual, SIDA y homofobia: estudio de casos con adolescentes pertenecientes a grupos religiosos. Tesis de licenciatura en Psicología, Universidad de Costa Rica, San José, Costa Rica.

Jiménez, José. (2014). Base de datos sobre textos LGBT aplicados al caso de Costa Rica, 1980-2013. Alajuela, inédito.

Koyama, Emi y Weasel, Lisa. (2002). From Social Construction to Social Justice: Transforming How We Teach about Intersexuality. Women's Studies Quaterly, 30(3,4), 169-178, Recuperado de http://www.jstor.org/ stable/40003252?seq $=2$

Kozak Rovero, Gisela. (2006). ¿Estudios sobre diversidad sexual, estudios sobre minorías sexuales? Revista venezolana de economía y ciencias sociales, 12(3), 135-141.

Madrigal Pana, Johnny. (1998). Al vaivén de un cabezal: un estudio sobre los traileros en América Central y su relación con el SIDA. Costa Rica: Instituto Latinoamericano y del Caribe de Planificación Económica y Social.

Madrigal, Allan y Gallo Curellas Ángela. (2000). Imagen de sí mismos de hombres travestis: estudio de casos cualitativo sobre la imagen de sí mismos de varios hombres que utilizan ropas femeninas en la ciudad de San José. Tesis de licenciatura en Psicología, Universidad de Costa Rica, San José, 
Marín Badilla, Liliana. (2004). Influencia del contexto sociocultural transmitido a través de personas significativas (familia nuclear y extensa) en la construcción e interpretación de las vivencias de dos mujeres que reconocen como lesbianas: análisis de casos. Tesis de licenciatura en Psicología, Universidad de Costa Rica, San José, Costa Rica.

Marín Esquivel, Rebeca. (2012). El tuteo en las comunidades homosexual y heterosexual herediana. Tesis de licenciatura en Lingüística, Universidad Nacional, Heredia, Costa Rica.

Mata, Alonso. (2009, 17 de mayo). Universidades promueven el respeto por la diversidad. La Nación. Recuperado de http://www.nacion.com/vivir/Universidades-promueven-respeto-diversidad_0_1049895039.html

Mata, Leonardo. (1988). ¿Cuán grande es la epidemia de SIDA en los hombres homosexuales de Costa Rica? Revista Costarricense de Ciencias Médicas, 9(3), 63-70.

Mata, Leonardo y Ramírez, Giselle. (1987). Consumo de drogas ilícitas por hombres homosexuales o bisexuales de Costa Rica, 1985-1986. Revista Costarricense de Ciencias Médicas, 9(3), 37-45.

Mata, Leonardo; Ramírez, Giselle y Rosero, Luis. (1987). Tipología y conducta de riesgo de infección con el virus de la inmunodeficiencia humana $(\mathrm{VIH})$, de hombres homosexuales de Costa Rica, 1985-1987. Revista Costarricense de Ciencias Médicas, 9(3), 21-35.

Menjívar Ochoa, Mauricio. (2007). Hombres inventados. Estudios sobre masculinidad en Costa Rica y la necesidad de nuevos supuestos para el cambio social. Diálogos Revista Electrónica de Historia, 8(1), 134-162. Recuperado de http://www.redalyc.org/articulo.oa?id=43980106

Molina, Iván. (2013) Profesionalización diferenciada. Composición y desempeño académico de los historiadores costarricenses. Revista A Contracorriente, 11(1), 110-151.

Muñoz Pinel, Alejandra María. (1998). Derecho a la no discriminación en la Ley General sobre el VIH-SIDA. Tesis de licenciatura en Derecho, Universidad de Costa Rica, San José, Costa Rica.

Murillo Rodríguez, Luis Guillermo. (2003). La tutela jurídica a las parejas formadas por personas del mismo sexo: uniones estables homoafectivas. Tesis de licenciatura en Derecho, Universidad de Costa Rica, San José, Costa Rica.

Lima, Lázaro. (2008). Deseos de estados queer en la producción crítica latina de los Estados Unidos. Revista Iberoamericana, 74(225), 959-971. 
López-Vicuña, Ignacio. (2004). Approaches to Sexuality in Latin America: Recent Scholarship on Gay and Lesbian Studies. Latin American Research Review, 39(1), 238-253. Recuperado de http://www.jstor.org/stable/1555391

Obando, Alexander. (2008). La gruta y el arcoíris: Antología de narrativa gay/ lésbica costarricense. San José: Editorial Costa Rica.

Ollé Méndez, Alberto. (2008). Vivencia del rechazo experimentado por personas transexuales: un estudio de caso. Tesis de licenciatura en Psicología, Universidad de Costa Rica, San José, Costa Rica.

Pérez Li, Ana Virginia. (2001). El derecho a la transexualidad: Propuestas para legislar los procesos de una cirugía de cambio de sexo. Tesis de licenciatura en Derecho, Universidad de Costa Rica, San José, Costa Rica.

Ramírez Arce, Silvia y Vargas Zúñiga, Marcia. (2007). Organización de la comunidad gay/lésbica costarricense por la defensa de sus derechos: configuración, desarrollo y alcances. Tesis de licenciatura en Psicología, Universidad de Costa Rica, San José, Costa Rica.

Renn, Kristen. (2010). LGBT and Queer Research in Higher Education: The State and Status of the Field. Educational Researcher, 39(2), 132-141, Recuperado de https://www.msu.edu/ renn/RennLGBTQueerEdResearcher.pdf

Rodríguez Pineda, Miguel Ángel. (1986). Cuantificación de linfocitos T en 37 varones homosexuales y su relación con prácticas sexuales e ingesta de drogas. Tesis de licenciatura en Microbiología, Universidad de Costa Rica, San José, Costa Rica.

Rodríguez, Eugenia. (2004). Bibliografía sobre estudios de las mujeres y de género en América Central, con énfasis en Historia (1957-2004). Diálogos Revista Electrónica de Historia, 5(1,2). Recuperado de http://revistas.ucr. ac.cr/index.php/dialogos/article/view/6256

Rostrán Saballos, Michele. (2012). Tecno-mujeres: una sistematización de la experiencia de dos mujeres transgénero con respecto al ejercicio de tres de sus derechos humanos fundamentales: derecho a la identidad, derecho a la expresión y derecho a vivir una vida libre de violencia. Tesis de licenciatura en Psicología, Universidad de Costa Rica, San José, Costa Rica.

Sandoval Solano, Luis Sandro. (2009). Dolor revestido de clandestinidad, experiencia de duelo en varones gays ante la pérdida por fallecimiento de su pareja afectiva, un estudio casuístico. Tesis de licenciatura en Psicología, Universidad de Costa Rica, San José, Costa Rica.

Schifter Sikora, Jacobo. (1989). La formación de una contracultura: homosexualismo y SIDA en Costa Rica. San José: Guayacán. 
Schifter Sikora, Jacobo y Madrigal Pana, Johnny. (1990). Primera encuesta nacional sobre SIDA: informe de resultados. San José: Instituto Latinoamericano y del Caribe de Planificación Económica y Social.

Schifter Sikora, Jacobo, Madrigal, J. y Aggleton, Peter. (1996). Bisexual communities and cultures in Costa Rica. En Aggleton, Peter. (Ed.). Bisexualities and AIDS: International Perspectives. Londres: Taylor and Francis.

Serrano Madrigal, Ester. (2002). De la memoria individual a la historia social: grupos de encuentro de las mujeres lesbianas costarricenses. Tesis de maestría en Estudios de la Mujer, Universidad de Costa Rica, San José, Costa Rica.

Soto López Graciela. (2008). El reconocimiento de la unión de hecho de parejas del mismo sexo. Tesis de licenciatura en Derecho, Universidad de Costa Rica, San José, Costa Rica.

Soto Rodríguez, Mario Andrés. (2013). Entre lo trans y lo tra(n)stornado: un análisis de la resignificación del diagnóstico recibido sobre la identidad de género en dos estudios de caso. Tesis de licenciatura en Psicología, Universidad de Costa Rica, San José, Costa Rica.

Suárez Reehag, Daría. (2000). Status legal de las lesbianas. Recuperado de: http://www.cipacdh.org/cipac_articulo_completo.php?art=56

Suárez Rehaag, Daría. (2008). jVisitas intra hospitalarias gais y lesbianas...si tenemos derecho! San José: Centro de Investigación y Promoción para América Central de Derechos Humanos.

Suárez Rehaag, Daria y Madrigal Ballestero, Francisco. (2000). Suicidio en la población homosexual costarricense. San José: Centro de Investigación y Promoción para América Central de Derechos Humanos.

Thayer, Millie. (1997). Identity, Revolution, and Democracy. Lesbian Movements in Central America. Social Problems, 44(3), 386-407, Recuperado de la base de datos Jstor.

Toro-Alfonso, José. (2005). El estudio de las homosexualidades: Revisión, retos éticos y metodológicos. Revista de Ciencias Sociales, 14, 78-97.

Trejos Camacho, Mario. (2008). Experiencias del activismo gay costarricense: La organización política como estrategia contra la discriminación. Tesis de licenciatura en Psicología, Universidad de Costa Rica, San José, Costa Rica.

Turner, Stephanie. (1999). Intersex Identities: Locating new intersections of sex and gender. Gender \& Society, 13(4), 457-479. 
Vargas Barrantes, Elida. (2013). Bases de la diferenciación sexual y aspectos éticos de los estados intersexuales. Revista Reflexiones, 92(1), 141-147.

Vargas Ruiz, Rodrigo. (2001). Pétalos y espinas: hombres gays, relaciones de pareja y violencia. Tesis de maestría en Antropología, Universidad de Costa Rica, San José, Costa Rica.

Vargas Ruiz, Rodrigo. (2003). Pétalos y espinas: hombres gays, relaciones de pareja y violencia. Argentina: Elaleph.

Vega Mirada, Lawrence. (2000). Antología de ensayos sobre textos literarios homoeróticos. Tesis de maestría en Literatura, Universidad de Costa Rica, San José, Costa Rica.

Weeks, Jeffrey. (2003). Sexuality. Londres: Routledge.

Wilson, Bruce M. (2007). Claiming individual rights through a constitutional court: The example of gays in Costa Rica. ICON, 5(2), 242-257. 